\title{
Análisis Crítico de las Medidas de Presión Propuestas para Mejorar la Formación Inicial de Docentes en Chile por el Panel de Expertos para una Educación de Calidad*
}

\author{
Critical Analysis of the Pressures for Improving Initial Teacher Education Proposed by \\ the Panel of Experts for a Quality Education in Chile
}

\begin{abstract}
Análise crítica das medidas de pressão propostas para melhorar a formação inicial de professores no Chile pelo painel de Especialistas para uma educação de qualidade
\end{abstract}

\section{Carmen Montecinos}

Pontificia Universidad Católica de Valparaíso, Escuela de Psicología.

Telf.: 562 322274366. Correo electrónico: carmen.montecinos@ucv.cl

\begin{abstract}
RESUMEN
En su informe del 9 de Julio 2010, el Panel de Expertos para una Educación de Calidad propone nueve recomendaciones para fortalecer la formación inicial de docentes en Chile. Seis recomendaciones están relacionadas con medición/accountability (presión) y otras tres proponen recursos para mejorar las condiciones en las carreras de pedagogía (apoyo). En este artículo se plantea que las propuestas de presión extienden hacia la formación inicial de docentes dispositivos ya instalados en el sistema escolar: estandarización, evaluación, competencia y accountability. Reconociendo los límites de las medidas de presión y sus sustentos ideológicos, se argumenta que las pruebas (PSU e INICIA) propuestas por el Panel deben estar sujetas al cumplimento de estándares y accountability. Respecto de la propuesta para la conformación de una red de campos pedagógicos, la evidencia muestra que se requieren políticas que otorguen sustentabilidad a la asociatividad entre instituciones de educación superior y del sistema escolar.
\end{abstract}

Palabras clave: formación inicial docente, nuevo management público, asociatividad universidad-sistema escolar, políticas educativas.

\begin{abstract}
The July 9th, 2010 report from the Panel of Experts for Quality Education offers nine recommendations to strengthen initial teacher education (ITE) in Chile. Six of them promote the use of tests at admissions and at graduation from a teacher education program (pressure) and the other three propose resources to improve the conditions for ITE (support). This article discusses how pressure measures extend devices already in use in the school system: standardization, evaluation, competition, and accountability. Acknowledging the limits of such pressure measures and their ideological underpinning, it is argued that the exams proposed (PSU and INICIA) must comply with evaluation standards and accountability. With respect to the recommendation regarding the development of a network of clinical schools, the evidence suggests the need of advanced policies that afford sustainability to partnerships between institutions of higher education and the school system.

Key words: initial teacher preparation, new public management, school-university partnerships, educational policies.
\end{abstract}

\footnotetext{
La autora agradece a FONDECYT (Proyecto No 1110505). Financiamiento adicional fue provisto a través del Centro de Investigación Avanzada en Educación (PIA-CONICYT, Project CIE-05).
} 


\section{RESUMO}

Em seu Informe de 9 de julho de 2010, o Painel de Especialistas para a Educação de Qualidade propõe nove recomendações para fortalecer a formação inicial de professores no Chile. Seis delas se relacionam com a medição/prestação de contas (pressão) e outras três propõem recursos para melhorar as condições nas carreiras de Pedagogia (apoio). No artigo, mostra-se que as propostas de pressão estendem-se à formação inicial de professores os dispositivos já instalados no sistema escolar: padronização, avaliação, competência e prestação de contas. Reconhecendo-se os limites de tais medidas de pressão e sua sustentação ideológica, argumenta-se que as avaliações (PSU e INICIA) propostas pelo Painel devem estar sujeitas ao cumprimento de padrões e prestação de contas. Com respeito às propostas para a conformação de uma rede de campos pedagógicos, evidência mostra que se requerem políticas que consentem sustentabilidade para a associação entre instituições de Ensino Superior-do sistema escolar.

Palavras chave: formação inicial docente, nova gestão pública, associação univerdidade-sistema escolar, políticas educativas.

\section{INTRODUCCIÓN}

En su informe del 9 de Julio de 2010, el Panel de Expertos para una Educación de Calidad (PEEC) propuso nueve recomendaciones para fortalecer la formación inicial de docentes (FID) en Chile. Estas abordan los dos ámbitos que han sido identificados como necesarios para apalancar el cambio en educación: presión y apoyo. Seis recomendaciones están relacionadas con medición/accountability (presión) y otras tres proponen recursos para mejorar las condiciones en las carreras de pedagogía (apoyo). A la fecha, Mayo 2014, las recomendaciones que se han traducido en proyectos de ley son, mayoritariamente, las medidas de presión. Es decir, regulaciones respecto del desempeño de los estudiantes de pedagogía en pruebas de ingreso (puntaje mínimo de 550 en la Prueba de Selección Universitaria, PSU $^{1}$ ), en pruebas de egreso (INICIA) y el fortalecimiento de la acreditación ${ }^{2}$. Para el PEEC, el punto de presión para apalancar el cambio es que las carreras de pedagogías reciban un mínimo de cuatro años de acreditación. Los recursos financieros que entrega el estado a las universidades y a sus estudiantes dependen de ello. El punto de presión, entonces, es el apoyo económico al cual los estudiantes de pedagogía pueden acceder para llegar a ser profesores.

Compartiendo la necesidad de contar con docentes de alta calidad, en este artículo se ofrece un análisis crítico de las medidas de presión propuestas en el informe del PEEC. Para contextualizar el análisis, en la primera sección se presentan las propuestas que son objeto de análisis. Luego se abordan algunas consideraciones generales respecto del uso de estándares y accountability como dispositivos de mejora. En la tercera sección se devela el sustento ideológico de las medidas de presión propuestas por el PEEC, Nuevo Management Público. Se observa que una política de mejoramiento basada en la evaluación debe contemplar dispositivos que aseguren que los instrumentos, y la interpretación de sus resultados, sean válidos y justos para todos los evaluados. Además, la evaluación de los egresados requiere medir el desempeño en tareas auténticas al trabajo docente. El PEEC

\footnotetext{
Proyecto Sobre Formación e Inicio del Ejercicio Docente. Última visita 21 de Mayo de 2014, recuperado des http://www. senado.cl/formacion-inicial-docente-comienza-la-discusion-en-general/prontus_senado/2014-01-22/204704.html. Si bien este proyecto deja abierta la posibilidad de usar otro indicador como ranking de notas en la enseñanza media, la política actual no considera la asignación de Becas Vocación de Profesor en base a ese ranking.

2 El informe consigna las medidas de apoyo que se han venido desarrollando en el marco de los proyectos financiados por el MECESUP. La última generación de estos proyectos son los Convenios de Desempeño adjudicados el 2013 a siete, y en el 2014 a cuatro, de las 56 instituciones de educación superior que forman profesores en Chile. Es decir mientras INICIA es obligatoria para las 56, los recursos de apoyo están disponibles para un grupo selecto de 11.
} 
espera que el sistema de accountability propuesto acorte la brecha entre los currículos que se imparten en las carreras de pedagogía y los requerimientos del sistema escolar. En la última sección se argumenta que abordar esta brecha requiere una estrategia más directa que implica el desarrollo de políticas que apoyen la asociatividad universidad-sistema escolar para el fortalecimiento simultáneo de la FID y de los centros escolares.

\section{EXIGENCIAS PROPUESTAS POR EL PEEC}

Luego de hacer un diagnóstico de las deficiencias en la FID en Chile, el Panel propone un conjunto de medidas que tienen "como principal objetivo, una formación inicial docente de la mayor calidad y exigencia posibles ,pero que, además, sea atractiva para los jóvenes con mayores habilidades iniciales" (Informe PEEC, 2010: 6). El listado de exigencias incluye la creación de un régimen de números derivados de exámenes y acreditación (Ball, 2010):

A todos los egresados de los programas de pedagogía se les exigirá aprobar un examen de habilitación para poder enseñar en la educación subvencionada por el Estado.

Los desempeños de los egresados de los diversos programas de pedagogía deben ser públicos. Estos deben ser considerados en el proceso de acreditación de los programas de formación inicial docente.

El examen deberá estar a cargo de un Consejo que otorgue garantías de imparcialidad y calidad del instrumento a todos los actores relevantes. El Panel estima indispensable que en un plazo no muy largo se desarrolle, a partir de la actual prueba INICIA, dicho examen de habilidades y que este sea pertinente a cada nivel y especialidad. (Informe PEEC, 2010: 6).

Además de abordar mediciones y sanciones asociadas al proceso y egreso de la formación inicial de profesores, se plantea la necesidad de aumentar la selectividad de quienes ingresan a las carreras de pedagogía. Es interesante que se le pida evidencia de su validez al sistema medición para el ingreso, pero no se le pida lo mismo a la medición de egreso:

Se postula que en un lapso prudente, se promueva que los programas de formación docente seleccionen a sus estudiantes de entre los estudiantes pertenecientes al 30 por ciento de mayores habilidades en el país. Si el instrumento elegido para definir este criterio fuese el promedio de la PSU, eso obliga a una exigencia de 555 puntos como mínimo. El Panel, en todo caso, estima que puede haber criterios complementarios o alternativos como el ranking de notas en la educación media, siempre que ellos demuestren capacidad de predecir rendimiento durante los estudios de pedagogía, resultados en el examen de habilitación o desempeño en el aula. (Informe PEEC, 2010: 59).

A través de INICIA, o su equivalente, este conjunto de exigencias instala en las carreras de pedagogía la tecnología de control que Carrasco (2013) señala ha sido desplegada a través del SIMCE en los centros escolares: es obligatorio para todas las instituciones de educación superior, sus resultados son públicos y serán usados por el Estado para asignar financiamiento, apoyos, incentivos y rankings. Resulta paradojal que mientras en Chile y en diversas partes del mundo hay un movimiento ciudadano y académico para disminuir las examinaciones estatales como dispositivo para mejorar los sistemas escolares, la "última generación" de políticas en Chile proponga expandir esta lógica a las universidades que forman profesores (Campos-Martínez y Fernández, 2014; Pino, 2014). 


\section{CONSIDERACIONES PRELIMINARES}

Las instituciones que forman profesores deben estar comprometidas con la mejora continua de sus procesos y de sus resultados. Los estándares orientadores, un sistema de examinación de las competencias profesionales de sus titulados y un sistema de accountability pueden ser dispositivos útiles para mejorar. Sin embargo, existe el riesgo que se transformen en dispositivos que perjudiquen antes que ayuden al objetivo de lograr que todos los estudiantes aprendan con calidad y equidad. Estos riesgos se asocian, en parte, a la definición de calidad docente que promueve la política pública, a una confusión entre estándares y estandarización y a la ausencia de una propuesta para un sistema de accountability democrático.

\section{1. ¿QUÉ ENTENDEREMOS POR UN PROFESOR DE CALIDAD?}

A la base del paquete de medidas de presión del PEEC hay una conceptualización respecto de calidad docente y desempeño docente que requiere ser problematizada. Es más, como lo han planteado Ávalos y Sotomayor (2012), entre otros autores, la formación de personas y producción de aprendizajes contienen la doble misión que configura la identidad de docentes en Chile. Esta identidad es tensionada por políticas de medición, incentivos y sanciones que reducen la tarea docente a la producción de resultados en las pruebas SIMCE. La introducción de las recomendaciones del PEEC incorpora estas tensiones a la FID.

Para la política pública, la calidad de la educación que ofrece un profesor se puede inferir solamente de los puntajes en las diversas pruebas del Sistema Nacional de Medición de la Calidad de la Educación (SIMCE). Diseñar un sistema de evaluación que fomente la calidad requiere ir más allá de instrumentos cuyos propósitos sean determinar la eficiencia y efectividad del trabajo docente (y carreras de pedagogía a través de la prueba INCIA ${ }^{3}$ ). En primer lugar, por profesor de calidad se entiende a un profesional con las competencias necesarias para generar prácticas profesionales individuales y colectivas que posibiliten a sus estudiantes beneficiarse de su participación en el centro escolar. Estos beneficios se refieren al desarrollo de sus capacidades académicas /intelectuales, sus habilidades sociales, artísticas y deportivas, al desarrollo de personas con altos niveles de bienestar subjetivo ${ }^{4}$, comprometidas con valores, actitudes y habilidades para participar como agentes en la construcción de una sociedad democrática.

Esta definición dista bastante de la definición propuesta por el instrumento Convenio de Desempeño, una iniciativa del MECESUP para fortalecer la formación inicial de profesores. Para la política pública, expresada en estos convenios, "se usará el concepto de calidad del titulado al que se asociarán los siguientes indicadores de resultado: a) Prueba Inicia (u otra prueba oficial que esté vigente al momento de egreso de las respectivas cohortes); b) Valor agregado producido por los nuevos profesores en los aprendizajes de sus alumnos en escuelas y liceos." Última visita 21 de Mayo de 2014, recuperado desde http://www.mecesup.cl/index2.php?id_portal=59 yid_seccion=3608 yid_contenido=15056.

$4 \quad$ Se refiere a la propuesta de pensar que el desarrollo de una sociedad no está solo en lo económico y material “(...) resignificar el horizonte del desarrollo poniendo al centro los proyectos de vida de las personas, es necesario ampliar la mirada y asumir en toda su complejidad una dimensión hasta ahora menos atendida del desarrollo, y de la cual el fenómeno de la felicidad forma parte: la subjetividad. Se entiende por subjetividad el espacio y el proceso en que los individuos construyen una imagen de sí, de los otros y del mundo en el contexto de sus experiencias sociales." (González, 2012: 16). 
En segundo término, las prácticas profesionales, siguiendo a Kemmis et al. (2012), contemplan la dimensión material-productiva expresada en las acciones y actividades propias del trabajo docente que buscan producir aprendizaje, así cómo cumplir con la misión socializadora de la educación formal. Además consideran la dimensión cultural discursiva (lo que se dice y se piensa) del trabajo docente así como los aspectos socialpolíticos, es decir, los tipos de relaciones, la solidaridad y dinámicas de poder entre los diferentes actores del espacio social en que se desarrolla la educación. El trabajo docente requiere de colaboración y colegialidad (Hargreaves y Fullan, 2012; Shirley et al., 2013). El uso de evaluaciones, sin embargo, promueve la competencia entre docentes o centros escolares como motor del mejoramiento (Pino, 2014).

\subsection{FORMULACIÓN DE ESTÁNDARES ORIENTADORES VERSUS ESTANDARIZACIÓN}

Para evaluar la calidad de los egresados de las carreras se formularon los Estándares Orientadores para las Carreras de Pedagogía (Ministerio de Educación, 2012). La existencia de estándares puede representar una oportunidad para evitar que los currículos se expresen como una acumulación de saberes fragmentados y descontextualizados, lo que ha sido característico en nuestro país. Los estándares pueden convocar al diálogo entre formadores para consensuar un currículo con coherencia interna y externa, posibilitar la deliberación respecto a cómo las demandas del desempeño laboral orientarán el trabajo de los formadores de profesores y ayudar a crear comunidad, particularmente si los docentes del sistema escolar son parte de esas conversaciones.

La experiencia nacional e internacional, sin embargo, alerta el riesgo que estas orientaciones se transformen en estandarización y prescripción de programas de asignaturas diseñados de manera centralizada, para su posterior implementación por los académicos universitarios. Se podría pensar que uniformar las metas, contenidos, estrategias de enseñanza y evaluaciones es una forma de asegurar igualdad de oportunidades entre universidades y entre secciones de una misma asignatura en una IES. Este enfoque, sin embargo, no permite a los futuros docentes observar y comprender el razonamiento pedagógico de sus profesores, no les permite entender cómo se contextualiza la enseñanza y el rol de la reflexión docente para la mejora continua. Es decir, no modela o desarrolla la docencia como una profesión. Por otra parte, igualdad de oportunidades implica diferenciación en la mediación pedagógica.

Los programas de asignaturas "a prueba de profesores" construidos por "expertos" ajenos al lugar donde se construye la relación pedagógica, posicionan al docente como un técnico. Este posicionamiento es particularmente problemático en la FID, considerando que está ampliamente documentado que los futuros profesores enseñan tal como se les enseñó, no como se les dijo que debían enseñar (Kennedy, 1999). Si el docente universitario modela el trabajo de un técnico, esta es la concepción de profesionalidad que aprenderán los futuros profesores. Este tipo de formación técnica puede ser instrumental a políticas como el Programa de Apoyo Compartido (PAC) que implementa el MINEDUC hasta $4^{\circ}$ año de educación básica, el cual entrega un currículo prescrito clase a clase. La visión del trabajo docente que sustenta programas como el PAC no da cuenta de la complejidad de enseñar a un grupo diverso de estudiantes, atendiendo simultáneamente y de manera contextualizada a múltiples demandas (Hammerness et al., 2005). Una FID de calidad, no obstante, se caracteriza por preparar a los profesionales para trabajar en la complejidad a través de la orquestación de diversas estrategias y conocimientos. 


\section{3. ¿QUIÉN DEBE RENDIR CUENTAS Y QUÉ CUENTAS RENDIR EN UN SISTEMA DE} "ACCOUNTABILITY"?

La propuesta del PEEC respecto a que las universidades que forman profesionales, en este caso profesores, den cuenta de sus procesos y resultados no es en sí objetable. Dar cuenta es parte del contrato social entre las IES que forman a los profesionales y la sociedad en la cual ejercerán (Schalock, 1998). Lo que hacen los profesores, y cuan bien lo hacen, impacta en el aprendizaje de sus estudiantes y su bienestar, por lo tanto, poder emitir juicios evaluativos que orienten el mejoramiento es potencialmente valioso. No obstante, la rendición de cuentas no puede tener como fin determinar quién puede o no acceder a becas y financiamiento para cumplir su vocación docente, como lo señala el informe PEEC. El fin último de un sistema de rendición de cuentas es el desarrollo de la responsabilidad con que cada profesor debe asumir su labor profesional (Schalock, 1998). Este fin pareciera estar ausente en los principios que dan coherencia al conjunto de propuestas.

A la base del paquete de medidas de presión del PEEC está el supuesto de que los niños, niñas y jóvenes chilenos aprenderán mejor porque sus profesores son objeto de un sistema de incentivos y castigos administrado por un Estado Evaluador (Carrasco, 2013; Martinic, 2010). El compromiso de los docentes con sus estudiantes, con la comunidad escolar y con su profesión, al parecer, no genera suficiente motivación por hacer bien el trabajo. Más premios o incentivos externos al trabajo docente escasamente desarrollarán las disposiciones profesionales asociadas a un buen desempeño; como es el compromiso con la equidad y la convicción que todos los estudiantes pueden aprender (Johnston et al., 2011).

Russell, Higgins y Raczek (2004) hacen notar una importante discrepancia entre la definición formal del concepto de accountability expresada en los diccionarios y la definición operacional expresada en las políticas educativas. La definición formal de accountability, refiere a que las personas investidas de responsabilidad deben dar cuenta, es decir, describir y explicar su gestión para poder determinar si esa gestión fue responsable. En el Diccionario Español-Inglés de la Universidad de Granada, España, accountability se traduce como: "responsabilidad en la gestión, rendición de cuentas, justificación, responsabilidad laboral, aprovechamiento de los recursos"5.

La definición formal, entonces, contrasta con la definición operacional expresada en políticas de examinación y rendición para las cuales "contar" se reduce al sentido matemático del término. Siguiendo a Ryan (2005), el cambio (fortalecimiento) se apalanca mejor a través de modelos democráticos de accountability que requieren que todos los actores, incluyendo a quienes diseñan la política, den cuenta de su responsabilidad por las discrepancias entre lo que ocurre y lo que se prometió debería ocurrir. En la propuesta PEEC, el modelo de accountability no solicita que los responsables del diseño de las políticas den una explicación a su comunidad de lo que hicieron o dejaron de hacer para mejorar la calidad de los profesores. Más aun, el PEEC no se pronuncia sobre los requisitos técnicos que deben demostrar los instrumentos de evaluación que se propician y sobre el sistema de accountablity que los regulará.

Ver en http://eubd1.ugr.es/xtf/search?lng=en ysort=title ykeyword=accountability 
Estudios Pedagógicos, vol. XL, Número Especial 1: 285-301, 2014 ANÁLISIS CRÍTICO DE LAS MEDIDAS DE PRESIÓN PROPUESTAS PARA MEJORAR LA FORMACIÓN INICIAL DE DOCENTES EN CHILE POR EL PANEL DE EXPERTOS PARA UNA EDUCACIÓN DE CALIDAD

\section{4. ¿CUÁLES SON LAS OBJECIONES AL ORIENTAR EL MEJORAMIENTO DE LA FORMACIÓN INICIAL DE PROFESORES A TRAVÉS DE UN “RÉGIMEN DE NÚMEROS"?}

\subsection{CONSIDERACIONES IDEOLÓGICAS}

La medición, estandarización y accountability representan una forma de gubernamentalidad basada en un tipo de conocimiento -régimen de números - a través del cual se concibe la mejora en calidad y eficiencia en los sistemas educativos (Ball, 2010; Ozga 2008 Cit., en Ball, 2010). En Chile, estos números surgen del programa PISA, TEDS-M, diversos ranking OECD, SIMCE, PSU e INICIA. Desde los años 80, los sucesivos gobiernos en Chile han ido intensificando este régimen de números a fin de monitorear, direccionar y reformar el sistema educativo. Comparaciones en el desempeño en estos indicadores numéricos hace a las naciones, instituciones educacionales y alumnos "legibles" para los gobiernos (Ball, 2010).

Esta aproximación es consistente con la adopción en el sector público de las prácticas de gestión del sector privado, lo que se conoce como el Nuevo Management Público (NMP) (Gunter, 2008). En Chile, las principales características del NMP definidas por Wittmann (2008) se ejemplifican en la privatización de la educación con proveedores privados que no están sujetos al estatuto docente, gestión de resultados, sistemas de rendición de cuentas basados en mediciones estandarizadas definidas por agentes externos y la competencia como incentivo al mejoramiento (Montecinos et al., 2013). Por ejemplo, el PEEC propone exigir el examen de habilitación solamente para poder enseñar en la educación subvencionada por el Estado, dejando fuera a los futuros docentes que se desempeñan en colegios particular pagados.

Con relación al trabajo docente, la lógica del NMP promueve sistemas de evaluación del desempeño según estándares y bonos dependientes de resultados que promueven identidades profesionales orientadas al emprendimiento (Sisto, Montecinos y Ahumada, 2013). Lo que hace la propuesta del PEEC es agregar otra evaluación a ese sistema, extendiendo su lógica a la construcción de identidades docentes en estudiantes universitarios quienes luego puedan estar más sensibles a naturalizar las políticas de medición y estandarización que regularán su trabajo en el sistema escolar.

Carrasco (2013), junto a otros autores, ha identificado evidencia que señala que estos dispositivos generan más consecuencias negativas que positivas en los procesos y condiciones de mejora del sistema escolar (Amrein y Berliner, 2002; Ball, 2010; Russell, Higgins y Raczek, 2004). Una vez instalados los sistemas de examinación en la formación de los futuros docentes, es probable que los efectos reseñados también se produzcan en las instituciones de educación superior:

Diversos estudios de países que han implementado este tipo de reformas durante los 90 y el 2000 convergen en concluir que este tipo de reformas contribuye a crear mayor desprofesionalización, segregación, aprendizajes mecánicos, desmoralización escolar, simplificación del currículo, desafección académica y cívica, logros de corto alcance, relocalización de los propósitos educacionales, autonomía ilusoria en las escuelas y un nuevo ambiente moral en educación que reconfigura los valores que una sociedad promueve, produce y transmite.

(Carrasco, 2013: 2). 
Estudios Pedagógicos, vol. XL, Número Especial 1: 285-301, 2014

ANÁLISIS CRIITICO DE LAS MEDIDAS DE PRESIÓN PROPUESTAS PARA MEJORAR LA FORMACIÓN INICIAL DE DOCENTES EN CHILE POR EL PANEL DE EXPERTOS PARA UNA EDUCACIÓN DE CALIDAD

\subsubsection{El estado evaluador}

Las propuestas del PEEC corresponden al "Estado Evaluador" que surge, siguiendo a Martinic (2010), cuando los mecanismos del modelo de mercado basado en la competencia entre IES no ofrecen los resultados prometidos. Para promover la rendición de cuentas en educación, Martinic (2010) señala que se requieren por lo menos cuatro condiciones: parámetros de calidad y estándares, instrumentos de evaluación e información, consecuencias para distintos actores y niveles y sistemas de autoridad. Estas condiciones están plasmadas en las exigencias propuestas por el PEEC cuando señala:

Un programa de estudios que obtenga menos de cuatro años de acreditación no podrá obtener beneficios económicos del Estado para sus estudiantes. Si ello ocurre por segunda vez, este programa no podrá aceptar nuevos estudiantes de pedagogía. Solo podrá volver a abrir sus puertas si en una tercera evaluación alcanza el umbral de cuatro años. Si el programa no logra esta acreditación, la institución que lo ofrece quedará impedida de ofrecer la carrera de pedagogía. (Informe PEEC, 2010: 7).

El "estado evaluador", en el contexto de las exigencias del PEEC, profundiza la lógica del NMP que concibe a la educación superior como un bien de consumo. El futuro profesor compró un servicio y si este no es de buena calidad, el proveedor del servicio, la IES, debe: "hacerse cargo obligatoriamente a su costo de la nivelación de sus egresados que, habiendo reprobado el examen de habilitación, aspiren a rendirlo por una segunda vez" (Informe PEEC, 2010: 6).

Esta exigencia supone que el profesor en formación no fue un agente activo de su proceso formativo. Es representado, más bien, como un producto manufacturado por la IES de la cual él o ella compraron un servicio. Más aún, ¿qué significa para el PEEC que los futuros docentes lleguen a entender su formación profesional como un proceso de nivelación? ¿Qué evidencias empíricas fundamentan el puntaje de corte que separa a quienes supuestamente tienen los conocimientos necesarios para crear oportunidades de aprendizaje de calidad a sus estudiantes y quienes no lo tienen?

\subsection{CONSIDERACIONES TÉCNICAS PARA LA INSTALACIÓN DE UN SISTEMA DE EVALUACIÓN}

Más allá de consideraciones ideológicas, dos aspectos técnicos requieren ser problematizados en el proceso de elaboración de pruebas de ingreso y de egreso como dispositivos para fortalecer la FID. Por un parte, para INICIA y la PSU es importante tener evidencias acerca de la validez de las posibles interpretaciones y uso que el PEEC propone dar a sus resultados. Un segundo aspecto, acotado a la prueba INICIA, refiere al tipo de evaluación más apropiada y a los conocimientos necesarios de medir para determinar si los titulados de las carreras de pedagogía logran los estándares. En un sistema de accountability democrático estos son aspectos sobre los cuales quienes serán sujetos a estas evaluaciones pedirán cuenta.

\subsubsection{Validez}

Una cosa es cierta en Chile, cada examen nacional para medir la efectividad y eficiencia del sistema educativo tiene como resultado develar las inequidades que dicho 
sistema reproduce. $\mathrm{Al}$ agregar INICIA al régimen de números esto ha vuelto a ocurrir ya que la distribución de resultados se asocia a la selectividad de las IES, la cual se asocia también al origen social de sus estudiantes. Consecuentemente, es imprescindible conocer las características psicométricas, en particular la validez de INICIA y de la PSU para los distintos grupos sociales que rinden esas pruebas. Por validez se entiende el conjunto de evidencias que avalan determinadas interpretaciones y usos de los resultados de estas pruebas, incluyendo evidencias de que su uso no genera consecuencias no deseadas sobre los evaluados (en este caso, la profesión docente) (Valverde, 2000).

Los estándares propuestos por el Comité Conjunto sobre Normas para la Evaluación en Educación (Joint Committee on Standards for Educational Evaluation) y los estándares propuestos por una comisión conjunta de la American Educational Research Association (AERA), American Psychological Association (APA) y el National Council for Measurement in Education (NCME) (2014), especifican un conjunto de prácticas que aseguran una interpretación válida y libre de sesgos de los resultados de evaluaciones educacionales. Implementar la prueba INICIA como una medición con consecuencias severas para los estudiantes de las carreras de pedagogía (poder conseguir empleo) y las carreras de pedagogía (acreditación), exige su ajuste a normas como las propuestas por el Comité Conjunto sobre Normas para la Evaluación de la Educación (Joint Committee on Standards for Educational Evaluation, 2010). Por ejemplo, el uso de INICIA y de la PSU tendría que responder a los Estándares para la Evaluación de Estudiantes definidos por ese comité. A modo de ilustración:

- Estándar P3 Acceso a la Información que Entrega la Evaluación: Se debe ofrecer acceso a la evaluación del estudiante, pero este acceso se debe limitar al estudiante y a otros legítimamente autorizados para ver esa información, tal que la confidencialidad se mantenga y la privacidad se proteja.

o No obstante, el Panel propone: "Los desempeños de los egresados de los diversos programas de pedagogía deben ser públicos. Estos deben ser considerados en el proceso de acreditación de los programas de formación inicial docente." (Informe PEEC, 2010: 6).

El conjunto de estándares asociados con las Normas de Precisión buscan asegurar que la información que produce la evaluación, respecto del aprendizaje y desempeño de los estudiantes, posibilite interpretaciones válidas y conclusiones justificadas:

- Estándar A3 Análisis Contextual: Variables del contexto y de los estudiantes que pueden influir en el desempeño deben ser identificadas y consideradas, así el resultado que obtiene el estudiante pueda ser interpretado válidamente.

o Se requiere que una evaluación orientada por estándares esté en estrecha relación con el currículo de las carreras de pedagogía. Es decir, que lo evaluado sea lo enseñado. Para interpretar los resultados de INICIA, siguiendo estos estándares, el informe para cada IES tendría que ofrecer un análisis del contexto (Valverde, 2000). Si el currículo no cubrió todo los contenidos evaluados, ¿qué se puede inferir acerca del estudiante examinado? y ¿qué sobre los programas?

- Estándar A7 Identificación y Manejo de Sesgos: La evaluación de los estudiantes debe estar libre de sesgos e modo que las conclusiones sean justas.

o Proponer un puntaje mínimo en la PSU como requisito de ingreso a las carreras de pedagogía es creer que el puntaje PSU es producto del mérito individual del postulante. La 
Estudios Pedagógicos, vol. XL, Número Especial 1: 285-301, 2014

ANÁLISIS CRIITICO DE LAS MEDIDAS DE PRESIÓN PROPUESTAS PARA MEJORAR LA FORMACIÓN INICIAL DE

DOCENTES EN CHILE POR EL PANEL DE EXPERTOS PARA UNA EDUCACIÓN DE CALIDAD

noción referida a que la PSU mide el mérito del estudiante ignora los resultados de estudios que muestran diferencias en los porcentajes de estudiantes de distintos estratos sociales que llegan a rendir la PSU. Luego, entre quienes la rinden, ignoran que el rendimiento se asocia al ingreso familiar y a la educación del padre (Contreras et al., 2007).

o Como sabemos, en Chile, los resultados PSU ordenan a los estudiantes que la rinden y la distribución de sus puntajes coincide estrictamente con la distribución del ingreso familiar de los estudiantes (Contreras, Corbalán, y Redondo, 2007). Para el año 2010 el promedio en la prueba de lenguaje de la PSU fue de 471,2 para egresados de colegios municipalizados, 502,8 y 605,4, respectivamente, para los egresados del sistema particular subvencionado y particular pagado ${ }^{6}$. Por otra parte, la distribución de puntajes PSU también es dispareja a lo largo de las distintas regiones de nuestro país, con los puntajes más altos tendiendo a concentrarse en la zona central del país.

Estos antecedentes sugieren que al usar la PSU para seleccionar a quienes serán los futuros profesores se corre el riesgo de avanzar hacia una homogeneización de esta fuerza laboral en función del origen social y oportunidades educativas. La investigación internacional muestra que es importante que los estudiantes de sectores marginados cuenten con profesores que pertenezcan y conozcan a las comunidades y reconozcan sus fortalezas. Los estudiantes ven en sus profesores modelos a seguir, y los profesores que comprendan sus circunstancias de vida pueden orientarlos respecto de cómo superar aquellas que limitan sus oportunidades.

En Chile, los datos muestran que profesores que se desempeñan en establecimientos educacionales que educan a estudiantes de menor nivel socioeconómico estudiaron en una mayor proporción en establecimientos municipales (Ortúzar, Flores y Milesi, 2012). Si los postulantes a profesores provenientes de centros escolares municipales decrecen como consecuencia de las políticas PEEC, ¿quién irá a enseñar en esos colegios, comprometiéndose con el proyecto educativo a largo plazo? Utilizar un puntaje de corte PSU de 550 o 555 puntos e INICIA requiere que la política se comprometa a desarrollar estudios que permitan monitorear y corregir cualquier tipo de inequidad que dichas medidas puedan generar.

\section{3. ¿QUÉ TIPO DE EVALUACIÓN SERÍA MÁS APROPIADA PARA INICIA?}

Para obtener un buen resultado en la prueba INICIA, con su foco en preguntas de respuesta cerrada en función al temario de contenidos definidos en los Estándares Orientadores para Profesores de Carreras de Pedagogía, no es necesario que los futuros profesores acudan a un centro escolar ${ }^{7}$. Los liceos y escuelas son lugares llenos de incertezas que demandan al profesional de la educación la capacidad para resolver problemas abiertos, con respuestas altamente contextualizadas e imbricadas en una ecología de prácticas institucionales a nivel del centro escolar, el sostenedor y la DEPROV, entre otros (Webster-Wright, 2009). Sin embargo, el tipo de respuestas que pide INICIA y la PSU implican solamente certezas.

6 Fuente: Compendio Estadístico Proceso de Admisión Año Académico 2010. Universidad de Chile. Última visita 12 de Mayo de 2014. Recuperado desde http://www.demre.cl/text/pdf/p2010/Compendio\%202010.pdf

7 Esta aseveración es refrendada por la alta correlación ( $\mathrm{R}$ Pearson=0,71) entre promedios institucionales PSU e INICIA formación profesional pedagógica y entre promedios PSU e INICIA formación disciplinaria ( $\mathrm{R}$ Pearson=0,88) en la evaluación INICIA 2010 a estudiantes de pedagogía en Educación Básica (García et al., 2011). Cuán bien le va a un futuro docente en INICIA-Disciplinaria depende más de lo que él o ella trajo a la universidad que de lo aprendido en su formación universitaria. 
En contraste al formato actual de la prueba INICIA, Linn y Herman (1997) plantean que el uso de evaluaciones educacionales orientadas por estándares incorporan nuevas formas de evaluación. Evaluar las capacidades de los futuros profesores para enseñar y para promover el aprendizaje en todos sus estudiantes requiere evaluar el desempeño en tareas "auténticas" al trabajo docente (ver por ejemplo la propuesta Muestras de Desempeño Docente un instrumento y procedimiento evaluativo desarrollado y validado en Chile por Montecinos et al., 2009, así como el modelo de evaluación que el MINEDUC implementa con docentes en servicio). En este tipo de evaluaciones se miden los conocimientos, habilidades y disposiciones profesionales que posibilitan a un docente mostrar su capacidad para pensar y actuar como un profesional de la educación.

Un examen a los futuros profesores no debería medir la capacidad para memorizar datos y hechos específicos o medir habilidades aisladas. INICIA tendría que incorporar tareas que (Linn y Herman, 1997):

1. son valiosas por sí mismas ya que promueven el aprendizaje.

2. modelan o ejemplifican el tipo de enseñanza que se espera en los profesores.

3. ontribuyen a la mejora, enfocándose en objetivos consistentes con los perfiles de egreso basados en competencias.

4. proporcionan mecanismos para el desarrollo profesional de los docentes evaluados.

5. involucran a los profesores en formación en actividades significativas que son intrínsecamente motivadoras.

6. conducen a una mayor y más apropiada responsabilización por el desempeño profesional.

\section{POTENCIAR POLÍTICAS PARA APOYAR LA ASOCIATIVIDAD IES-SISTEMA ESCOLAR PARA LA FORMACIÓN INICIAL DE PROFESORES}

Las políticas para mejorar la formación docente que han sido analizadas hasta ahora buscan apalancar el cambio mediante presiones externas a las universidades. Otra vía, esbozada en el informe del PEEC, es diseñar estrategias que posibiliten intensificar la colaboración entre las carreras de pedagogía y los centros escolares. El fin de la asociatividad universidad-sistema escolar es el fortalecimiento mutuo al compartir el rol de co-formadores de los futuros docentes.

Esa sugerencia recoge el consenso nacional e internacional respecto a que una parte importante del proceso de aprender a enseñar necesita ser desarrollado en los centros escolares (Childs, Edwards, y McNicholl, 2013; Hammerness et al., 2005; National Council for Accreditation of Teacher Education (NCATE), 2010). En su actual formulación, sin embargo, la recomendación queda corta para responder a las necesidades identificadas por la investigación nacional e internacional respecto a las condiciones que posibilitan una formación práctica de calidad (Foster et al., 2010; Montecinos, Walker y Cortez, aceptado; Zeichner, 2010).

\subsection{TENSIONES EN LA VINCULACIÓN IES-SISTEMA ESCOLAR}

El trabajo conjunto y la responsabilización compartida, en igualdad de estatus y poder, entre formadores de profesores con base en las IES y los formadores que trabajan en los centros escolares han demostrado ser complejos (Zeichner, Payne y Brayko, 
2012). Sin embargo, es a través de un trabajo conjunto que es posible definir una agenda de transformación mutua al servicio de una educación con equidad y calidad para todos (Darling-Hammond, 2006; Musset 2010; NCATE, 2010).

En una serie de estudios que recientemente realizamos con la participación de cinco universidades (13 carreras de pedagogía) distribuidas en distintas regiones de Chile se demostró la importancia de desarrollar políticas que den sustentabilidad a una mayor participación del sistema escolar en la FID $^{8}$. Algunos de los principales resultados serán resumidos a continuación, ilustrando cuáles podrían ser los temas para una agenda de transformación mutua a través de una red como la que propone el PEEC.

\subsubsection{Estudio 1}

Docentes directivos de 91 centros de práctica para las carreras de pedagogía participantes respondieron una encuesta en la cual se les pedía señalar los principales beneficios que esperaban obtener al colaborar con la formación inicial de docentes (Montecinos et al., 2013). Los 172 directivos, de manera casi unánime, señalaron que les interesaba que sus centros escolares participaran de la formación práctica ya que querían contribuir a la preparación de los nuevos docentes. Más allá de este fin altruista, también esperaban obtener ciertos beneficios para sus estudiantes y docentes.

Entre los beneficios, lo más frecuente fue señalar que se esperaba que la presencia de los practicantes generara oportunidades para: (a) acceder a más recursos humanos para apoyar a sus estudiantes (79\%), (b) mejorar el aprendizaje de los estudiantes del centro escolar (49\%) y apoyar la actualización y el desarrollo profesional de los docentes (45\%). Por otra parte, solamente un $36 \%$ señaló que a través de las prácticas se podrían fortalecer la implementación del plan de mejoramiento del centro escolar.

\subsubsection{Estudio 2}

En dicha encuesta, los docentes directivos también documentaron los obstáculos percibidos para lograr los beneficios esperados (Montecinos, Walker y Cortez, aceptado). La mayoría de los obstáculos identificados eran responsabilidad de las IES (62\%), con $34 \%$ de ellos atribuidos al centro escolar y solo un $4 \%$ que fue entendido como problemas asociados a la falta de vinculación entre ambas instituciones. Entre los obstáculos atribuidos a las IES lo más frecuente era la percepción del insuficiente nivel de competencias pedagógicas, disciplinarias y profesionales que presentan los practicantes para asumir las tareas requeridas por los centros escolares. Además, se cuestionó el currículo de práctica en cuanto al tiempo de permanencia de los practicantes en el centro escolar y la poca pertinencia de las tareas que se les pedía realizar. La insuficiente supervisión desde la universidad impedía que los docentes del centro recibieran información y formación adecuada para la tarea de mentor de los practicantes.

Aquí cabe señalar que el currículo de formación práctica es muy heterogéneo, involucra las prácticas iniciales que posicionan al practicante como observador hasta las prácticas profesionales que lo posicionan como profesor. Por lo tanto, las debilidades pueden obedecer

\footnotetext{
Proyecto FONDECYT No 1110505 "La contribución de los centros de práctica en la formación inicial docente: Perspectivas de docentes de aula y docentes directivos". C. Montecinos y H. Walker, co-investigadores.
} 
a juicios que se emiten sobre estudiantes de pedagogía en prácticas iniciales o intermedias a quienes los centros pueden pedir intervenciones para los cuales no han sido aún preparados.

Con respecto a los obstáculos atribuidos al centro escolar, lo más frecuente fue mencionar las mayores exigencias laborales para el profesor que recibía un practicante en su aula. Con menor frecuencia se planteó que los docentes no disponían de tiempo para apoyar la formación del practicante y la reticencia de algunos/as para asumir esta función. Con respecto a los obstáculos atribuidos a instancias de vinculación, se hizo referencia a la ausencia de estructuras organizacionales y una cultura de colaboración que posibilitaran un intercambio sistemático entre las IES y los centros escolares. La ausencia de intercambio y coordinación se asoció a que no siempre se lograba continuidad entre las propuestas metodológicas y pedagógicas del centro y las que propician las carreras de pedagogía.

\subsubsection{Estudio 3}

A través de una encuesta online supervisores de práctica $(\mathrm{N}=56)$ de las 13 carreras de pedagogía señalaron las principales dificultades que encontraban para implementar las prácticas en los centros escolares. Un 52\% de los obstáculos fue atribuido al centro escolar, como por ejemplo, la falta de flexibilidad de la programación escolar para acoger las tareas de los practicantes. Un 36\% de obstáculos fue atribuido a los docentes de aula que reciben practicantes. Entre este grupo de respuestas lo más frecuente fue mencionar la insuficiente disposición que mostraban para aceptar las innovaciones que los practicantes de pedagogía traían a la sala de clases. En este estudio, el rol que las universidades podrían tener en la generación de estos obstáculos fue minimizado.

\subsubsection{Estudio 4}

Sesenta y dos docentes del sistema escolar trabajando como mentores de practicantes participaron en entrevistas grupales. Ellos se distribuían en 11 centros escolares ubicados en cinco ciudades de Chile, representando distintas dependencias. A partir de estas entrevistas se logró construir una narrativa alternativa a la construida por el grupo de supervisores participando en el Estudio 3. Desde la perspectiva de los docentes mentores, las innovaciones propuestas desde las IES no siempre eran factibles:

Generalmente el profesor de la universidad pide una planificación constructivista, que planifiquen de esa manera, pero esas clases no le resultan a los practicantes (...). El no entiende que los alumnos no responderán a ese tipo de clases. Si no han venido a observar a los estudiantes de mi curso, ellos no pueden decirle al practicante como planificar su clase. (Docente de educación media, liceo municipal técnico-profesional).

\subsection{ORIENTACIONES}

Como ya lo han señalado Edwards y Mutton (2007), el cruce de acusaciones entre las IES y los centros escolares que atraviesan al practicante refleja contradicciones propias de ubicar la formación docente en un contexto que ha sido diseñado para la enseñanza de niños y jóvenes. Estas contradicciones se abordan a través de conversaciones que van más allá de programas de capacitación o enviando a practicantes a los centros escolares más tempranamente. Lo anterior implica ir más allá de lo recomendado por el PEEC al respecto: 
Estudios Pedagógicos, vol. XL, Número Especial 1: 285-301, 2014

ANÁLISIS CRIITICO DE LAS MEDIDAS DE PRESIÓN PROPUESTAS PARA MEJORAR LA FORMACIÓN INICIAL DE DOCENTES EN CHILE POR EL PANEL DE EXPERTOS PARA UNA EDUCACIÓN DE CALIDAD

la consolidación de redes de escuelas, liceos y colegios como "campos de práctica profesional", muy en línea con lo que ocurre con la formación en medicina. El Panel cree que no puede permitirse la acreditación de programas de pedagogía sin su correspondiente red de establecimientos que permita a los futuros profesionales tomar contacto con la sala de clases desde los inicios de su formación. (Informe PEEC, 2010: 7).

Es necesario, también, que los profesores universitarios participemos en esas aulas, así también que los docentes y docentes directivos de los centros escolares de dicha red participen en la definición del currículo de formación práctica (Beck y Kosnik, 2002; Montecinos y Walker, 2010). Los resultados de los cuatro estudios orientan el tipo de tareas que los practicantes necesitan realizar en los centros escolares para contribuir al logro de los beneficios esperados. Además, señalan la importancia de considerar las expectativas de los centros escolares que asumen el rol de co-formadores de los futuros docentes. Cabe preguntar cuáles son las instancias y estructuras creadas por las IES y por los centros escolares para abordar estos temas de manera conjunta.

Es de suponer que los miembros del Panel han considerado que la formación inicial de médicos es mucho más cara que la formación de docentes, en parte, por la calidad de los campos clínicos y la extensa especialización de los profesionales que forman a los futuros médicos. Tener una formación inicial de docentes con un componente clínico como ocurre en medicina, supone recursos que la política pública tendría que contemplar y/o subir sustantivamente el costo actual de las carreras de pedagogía. Si los médicos que forman parte del staff de los campos clínicos son remunerados, también debería remunerarse el trabajo de los docentes en el staff de los campos clínicos de las carreras de pedagogía. Por otra parte, la política de carrera docente debería incluir el rol que los docentes experimentados y con buen desempeño cumplen en la formación práctica de los estudiantes de pedagogía; aspecto ausente del proyecto de ley presentado por el gobierno del presidente Piñera.

\section{CONCLUSIONES}

Mejorar la formación docente en Chile es una prioridad nacional y también lo es para las IES que forman profesores. En este artículo, por una parte se han criticado las medidas de presión que propuso el Panel de Expertos para una Educación de Calidad, considerando que se han traducido en proyectos de ley que merecen la atención de las IES para influir en su diseño. Dichas propuestas, si bien son populares con quienes diseñan políticas inspiradas por el Nuevo Management Público, contradicen lo que las investigaciones muestran respecto de su efectividad para lograr mejoras sustantivas en la calidad y equidad de las oportunidades de aprendizaje que tienen los estudiantes en el sistema escolar. Utilizar como principal mecanismo de mejoramiento de la FID instrumentos de medición que han demostrado tener sesgos de clase social es muy probable que contribuya a perpetuar los altos índices de inequidad en nuestro sistema educativo.

Por otra parte, se ofrecen tres propuestas para fortalecer este mecanismo. Primero, desde las IES y desde los propios estudiantes de pedagogía se requiere exigir que las evaluaciones que instale la política pública se ajusten a lo que la comunidad internacional define como buenas prácticas en evaluación de programas y estudiantes. Segundo, fortalecer el sistema 
Estudios Pedagógicos, vol. XL, Número Especial 1: 285-301, 2014 ANÁLISIS CRÍTICO DE LAS MEDIDAS DE PRESIÓN PROPUESTAS PARA MEJORAR LA FORMACIÓN INICIAL DE DOCENTES EN CHILE POR EL PANEL DE EXPERTOS PARA UNA EDUCACIÓN DE CALIDAD

de accountability requiere incorporar a los distintos involucrados en la FID, no sólo a los estudiantes de pedagogía o las IES que los preparan para el ejercicio laboral. Tercero, la recomendación del PEEC respecto a la creación de redes de centros escolares para el desarrollo de la formación práctica requiere que la política pública considere recursos para apalancar el fortalecimiento de la asociatividad IES - sistema escolar. La investigación nacional e internacional señala que esta es una alianza compleja que sobrepasa voluntades institucionales (NCATE, 2010).

\section{REFERENCIAS BIBLIOGRÁFICAS}

American Educational Research Association, American Psychological Association y National Council for Measurement in Education. (2014). Standards for educational and psychological testing. Washington DC: American Psychological Association.

Amrein, A.L., \& Berliner, D.C. (2002). High-stakes testing, uncertainty, and student learning. Education Policy Analysis Archives, vol.10, n.18. Última visita 14 de abril de 2014. Recuperado desde http://epaa.asu.edu/epaa/v10n18/

Ávalos, B. y Sotomayor, C. (2012). Cómo ven su identidad los docentes chilenos. Perspectiva Educacional, vol.51, n.1, 57-86.

Ball, S.J. (2010). New voices, new knowledges and the new politics of education research: the gathering of a perfect storm? European Educational Research Journal, vol.9, n.2, 124-137.

Beck, C., \& Kosnik, C. (2002). Professors and the practicum: involvement of university faculty in preservice practicum supervision. Journal of Teacher Education, vol.53, n.1, 6-19.

Campos-Martínez, J. y Fernández, M.B. (2014). Resistiendo la emboscada neoliberal desde las escuelas: experiencias de organización docente contra las pruebas estandarizadas. Docencia, vol.52, 73-87.

Carrasco, A. (2013). Mecanismos performativos de la institucionalidad educativa en Chile: pasos hacia un nuevo sujeto cultural. Observatorio Cultural, vol.15, artículo 1.

Childs, A., Edwards, A., \& McNicholl, J. (2013). Developing a multi-layered system of distributed expertise: what does cultural historical theory bring to understandings of workplace learning in school-university partnerships? In O. McNamara, J. Murray, \& M. Jones (Eds.), Workplace learning in teacher education (pp. 29-46). Dordecht: Springer.

Contreras, M., Corbalán, F. y Redondo, J. (2007). Cuando la suerte está echada: estudio cuantitativo de los factores asociados al rendimiento en la PSU. Última visita 10 de abril de 2014. Recuperado desde http://www.opech.cl/bibliografico/calidad_equidad/Estudio_sobre_la_PSU_ Contreras_Corbalan_Redondo.pdf

Darling-Hammond, L. (2006). Constructing 21st-century teacher education. Journal of Teacher Education, vol.57, n.3, 300-314.

Edwards, A., \& Mutton, T. (2007). Looking forward: rethinking professional learning through partnership arrangements in initial teacher education. Oxford Review of Education, vol.33, n.4, 503-519.

Foster, R., Wimmer, R., Winter, N., \& Snart, F. (2010). Field experiences in teacher education: what is and what could be - a case study of the University of Alberta. In T. Falkenberg, \& H. Smits (Eds.), Field experiences in the context of reform of Canadian teacher education programs (Vol. 2) (pp. 187-224). Winnipeg, Canada: Faculty of Education of the University of Manitoba. Última visita 11 de marzo de 2014. Recuperado desde http://www.umanitoba.ca/education

García, M.J., Lacerna, P., Manzi, J., Meckes, L., Ortega, L., Pavez, P., Ramos, I. y San Martín, E. (2011). ¿Qué características de la formación inicial docente se asocian a mayores avances en su aprendizaje de conocimientos disciplinarios y pedagógicos? Trabajo presentado en el Coloquio MIDE-UC. Santiago, Chile. Última visita 13 de abril de 2014. Recuperado desde http://mideuc.cl/ 
Estudios Pedagógicos, vol. XL, Número Especial 1: 285-301, 2014

ANÁLISIS CRIITICO DE LAS MEDIDAS DE PRESIÓN PROPUESTAS PARA MEJORAR LA FORMACIÓN INICIAL DE DOCENTES EN CHILE POR EL PANEL DE EXPERTOS PARA UNA EDUCACIÓN DE CALIDAD

wp-content/uploads/2011/11/Caracteristicas-programas-formacion-inicial-docente-11_2011.pdf

González, P. (Coordinador). (2012). Informe sobre Desarrollo Humano en Chile 2012, Bienestar subjetivo: el desafío de repensar el desarrollo. Santiago, Chile: Programa de las Naciones Unidas para el Desarrollo, PNUD.

Gunter, H. (2008). Policy and workforce reform in England. Educational Management Administration \& Leadership, vol. 36, n.2, 253-270.

Hammerness, K., Darling-Hammond, L., Bransford, J., Berliner, D., Cochran-Smith, M., McDonald, M., \& Zeichner, K. (2005). How teachers learn and develop. In L. Darling-Hammond, \& J. Bransford (Eds.), Preparing teachers for a changing world: what teachers should learn and be able to do (pp. 358-389). Indianapolis, IN: Jossey-Bass.

Hargreaves, A., \& Fullan, M. (2012). Professional capital. transforming teaching in every school. New York \& Toronto: Teachers College Press. Ontario Principals' Council.

Johnston, P., Almerico, G., Henriott, D., \& Shapiro, M. (2011). Descriptions of dispositions for assessment in pre-service teacher education field experiences. Education, vol.132, n.2, 391-402.

Kemmis, S., Edwards-Groves, C., Wilkinson, J., \& Hardy, I. (2012). Ecologies of practices. In P. Hager, A. Lee, \& A. Reich (Eds.), Practice, learning and change (pp. 33-50). Dordecht: Springer.

Kennedy, M. (1999). The role of preservice teacher education. In L. Darling-Hammond, \& G. Sykes (Eds.), Teaching as the learning profession: handbook of policy and practice (pp. 54-85). San Francisco: Jossey-Bass.

Linn, R.L., \& Herman, J.L. (1997, February). Standards-led assessment: Technical and policy issues in measuring school and student progress. CSE Technical Report 426. National Center for Research on Evaluation, Standards, and Student Testing (CRESST). Center for the Study of Evaluation, Graduate School of Education and Information Studies, University of California, Los Angeles.

Martinic, S. (2010). Cambios en las regulaciones del sistema educativo. ¿Hacia un estado evaluador? En S. Martinic y G. Elacqua (Eds.), ¿Fin de ciclo? Cambios en la gobernanza del sistema educativo (pp. 55-80). Santiago, Chile: Facultad de Educación de la Pontificia Universidad Católica de Chile y la Oficina Regional para América Latina de UNESCO.

Ministerio de Educación. (2012). Estándares orientadores para egresados de carreras de pedagogía en educación básica. Santiago, Chile: Ministerio de Educación.

Montecinos, C., Walker, H. y Cortez, M. (en imprenta). Sugerencias de docentes directivos para mejorar la formación práctica en las carreras de pedagogía: transitando de acciones fragmentadas a una participación legítima en los colegios. Estudios Pedagógicos.

Montecinos, C., Walker, H., Cortez, M. y Maldonado, F. (2013). Beneficios para los centros escolares que colaboran en la formación inicial docente: perspectivas de docentes directivos. Páginas de Educación, vol.6, n.1, 31-53.

Montecinos, C. y Walker, H. (2010). La colaboración entre los centros de práctica y las carreras de pedagogía. Docencia, vol.42, 65-73.

Montecinos, C., Solís, M.C., Contreras, I. y Rittershaussen, S. (2009). Muestras de Desempeño Docente: un instrumento para evaluar la calidad de la enseñanza y su impacto en el aprendizaje. Santiago, Chile: Ediciones Universidad Católica de Chile.

Musset, P. (2010). Initial teacher education and continuing training policies in a comparative perspective: Current practices in OECD countries and a literature review on potential effects. OECD Education Working Papers, N.48. OECD Publishing. doi: 10.1787/19939019

National Council for Accreditation of Teacher Education (NCATE) (2010). Transforming teacher education through clinical practice: A national strategy to prepare effective teachers. Report of the Blue Ribbon Panel on Clinical Preparation and Partnerships for Improved Student Learning. Washington, DC: NCATE. Última visita 5 de marzo de 2011. Recuperado desde: http://www.ncate. org/LinkClick.aspx?fileticket=zzeiB1OoqPk\%3D ytabid=715

Ortúzar, S., Flores, C. y Milesi, C. (2012). Distribución de profesores principiantes, jefes UTP $y$ directores en distinto tipo de establecimientos: variación en sus características educacionales y 
percepciones. Trabajo Presentado en el Segundo Congreso Interdisciplinario de Investigación en Educación, Santiago, Chile.

Panel de Expertos para una Educación de Calidad. (2010). Informe Final: Primera Etapa. Propuestas para fortalecer la profesión docente en el sistema escolar chileno. Santiago, Chile: Ministerio de Educación.

Pino, M. (2014). Los valores que sustentan el Simce: pensando en un sistema de evaluación más allá de una medición estandarizada. Docencia, vol.52, 15-29.

Russell, M., Higgins, J., \& Raczek, A. (2004). Accountability, California style: counting or accounting? Teachers College Record, vol.106, n.11, 2102-2127.

Ryan, K.E. (2005). Making educational accountability more democratic. American Journal of Evaluation, vol.26, n.4, 532-543.

Schalock, M.D. (1998). Accountability, student learning, and the preparation and licensure of teachers: Oregon's teacher work sample methodology. Journal of Personnel Evaluation in Education, vol.12, n.3, 269-286.

Shirley, D., Fernández, M.B., Parra, M.O., Berger, A. y Borba, G. (2013). La cuarta vía de liderazgo y cambio en América Latina: perspectivas en Chile, Colombia y Brasil. Pensamiento Educativo. Revista de Investigación Educacional Latinoamericana, vol.50, n.2, 5-27.

Sisto, V., Montecinos. C. y Ahumada, L. (2013). Disputas de significado e identidad: la construcción local del trabajo docente en el contexto de las políticas de evaluación e incentivo al desempeño en Chile. Universitas Psychologica, vol.12, n.1, 173-184.

Valverde, G.A. (2000). La interpretación justificada y el uso apropiado de los resultados de las mediciones de logro. En P. Ravela (Ed.), Los próximos pasos: ¿Hacia dónde y cómo avanzar en la evaluación de aprendizajes en América Latina? (pp. 21-30). Santiago de Chile: PREAL.

Webster-Wright, A. (2009). Reframing professional development through understanding authentic professional learning. Review of Educational Research, vol.79, n.2, 702-739.

Wittmann, E. (2008). Align, don't necessarily follow. Educational Management Administration \& Leadership, vol.36, n.1, 33-54.

Zeichner, K. (2010). University-based teacher education. Journal of Teacher Education, vol.61, n.1-2, 89-99.

Zeichner, K., Payne, K., \& Brayko, K. (2012). Democratizing knowledge in university teacher education. Última visita 12 de Junio de 2012. Recuperado desde http://ccte.org/wp-content/pdfsconferences/ccte-conf-2012-fall-zeichner-democratizing-knowledge.pdf 
